\title{
The Kanehira-Hatusima 1940 Collection of New Guinea Plants. XVII.
}

\author{
By
}

\section{R. Kanehira and S. Hatusima}

Received October $2,1949$.

\section{R. Kanehira \& S. Hatusima: Dilleniaceae.}

?Dillenia philippinensis var. pubiflora Merr. Enum Philip. Fl. Pl. 3 (1923) 61.

No. 13234 Kanehira-Hatusima, Waren March 27, 1940. In high rainforests at $2 \mathrm{~m}$. altitude.

A very large tree up to $40 \mathrm{~m}$. in height and $1.5 \mathrm{~m}$. in diameter, bark reddish.

Distrib. Philippines. A new addition to the flora of New Guinea.

Hibbertia novo-guineensis GIBBS, Contrib. Phytog. \& Fl. Arfak Mts. (1917) 148.

Nos. 14054, 13487 Kanehira-Hatusima, Angi, Arfak Mts., April 5-9, 1940. In edge of low spinneys on the summit of Mt. Koebre at $2200 \mathrm{~m}$. altitude; scandent.

Distrib. Endemic; the type was from Angi.

Teracera moluccana Marteldi in Becc. Malesia 3 (1886) 153; Diels in ENGL. Bot. Jahrb. 57 (1922) 440.

No. 12403 Kanehira-Hatusima, Patema, Nabire, March 6, 1940. In edge of rain-forests at $300 \mathrm{~m}$. altitude. No. 11582 Kanehira-Hatusima, Nabire, Feb. 25, 1940. In edge of secondary forests at $2 \mathrm{~m}$. altitude; a seandent shrub, up to $5 \mathrm{~m}$. high.

Distrib. Amboina.

R. Kanehira \& S. Hatusima: Actinidiaceae.

§etosae.

Saurauia ( $\S$ Setosae) angica Kanehira et Hatusima, sp. nov. Fig. 1.

Frutex ad $3 \mathrm{~m}$. altus, rami ramulique teretes, setosi, brevissimi (vix $1 \mathrm{~mm}$. longi) dense vestiti. Folia parva, obovato-elliptica ad obovato- 


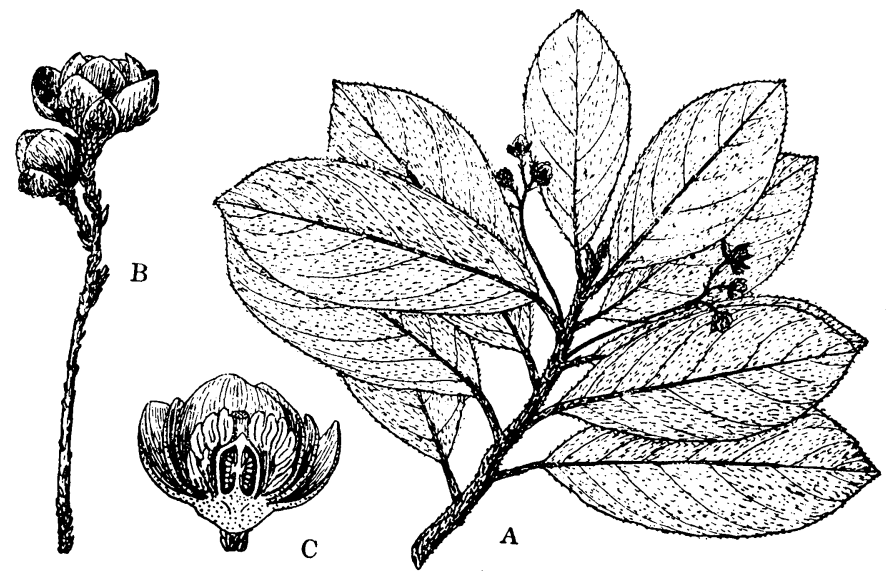

Fig. 1. Saurauia angica KAN. et HAT. (No. 13851)

A Branchlet with flowers $\times 2 / 3$. B Inflorescence $\times 2 . \quad$ C Flower in $1 . s . \times 3$.

oblonga, chartacea, $3-5 \mathrm{~cm}$. longa, $1.5-2.3 \mathrm{~cm}$. lata, apice acuta, basi obtuse rotundata, margine integra spinulosa, utrinque adpresse fusco-hirta, supra in sicco fusco-brumnea, subtus pallida, nervi laterales 6 vel 7 , arcuatim adscendentes; petiolo $0.5-1 \mathrm{~cm}$. longo setoso. Inflorescentiae axillares, pedunculatae, saepius 3 -florae, pedunculi circ. $1.5 \mathrm{~cm}$. longi sparse setulosi, pedicelli 5-11 mm. longi, densiuscule setulosi, bracteolati, bracteolis oblongolanceolatis cire. $1.5 \mathrm{~mm}$. longis dorso setulosis. Flores in alabastro cire. $5 \mathrm{~mm}$. diametro; sepala ovato-orbicularia inaequiformia, 2 exteriora minora extus sparse setulosa, 3 interiora majora circ. $5 \mathrm{~mm}$. longa dorso tantum sparsissime setulosa; petala 5 , ovato-elliptica circ. $4 \mathrm{~mm}$. longa glabra; stamina numerosa, antherae cire. $1 \mathrm{~mm}$. longae; ovarium glabrum, stylo 1 , circ. $1 \mathrm{~mm}$. longo.

No. 13851 Kanehira-Hatusima, Angi, Arfak Mts., April 8, 1940. In mossy forests, Iray, Lake Giji at about $1900 \mathrm{~m}$. altitude.

This is well characterized by its small leaves and simple styles. In its small leaves, this may be contrasted with Saurauia calyptrata LautB.

Saurauia bifida WARB. in Engl. Bot. Jahrb. 13 (1891) 380; K. Schum. et Lautb. Fl. Deutsch. Schutzg. Süds. (1901) 445 ; Diels in Engl. Bot. Jahrb. 57 (1922) 448.

No. 11879 Kanehira-Hatusima, Chaban, Nabire, Feb. 28, 1940. In rain-forests at $300 \mathrm{~m}$. altitude. A shrub $2.5 \mathrm{~m}$. in height, flowers white. Distrib. Endemic; north-eastern New Guinea.

Saurauia ( $\S$ Setosae) momiensis Kanemira et Hatusima sp nov. Fig. 2. Frutex circ. $5 \mathrm{~m}$. altus, ramuli dense fusco-hispidi. Folia oblongo- 
oblanceolata vel late oblanceolata, chartacea, apice breviter acuminata, basi angustata, margine setoso-serrata, $12-17 \mathrm{~cm}$. longa, $4.5-6.5 \mathrm{~cm}$. lata, utrinque sparse hispida, nervi laterales 12 vel 13, ad prope marginem arcuatim adscendentes, ut costa subtus hirti ; petiolo circ. $1.5 \mathrm{~cm}$. longo, dense hispido Inflorescentiae axillares saepe triflorae, circ. $2-3 \mathrm{~cm}$. longae, pedunculis $1-1.5 \mathrm{~cm}$. longis, pedicelli eirc. $5 \mathrm{~mm}$. longi ut pedunculis dense setosi,

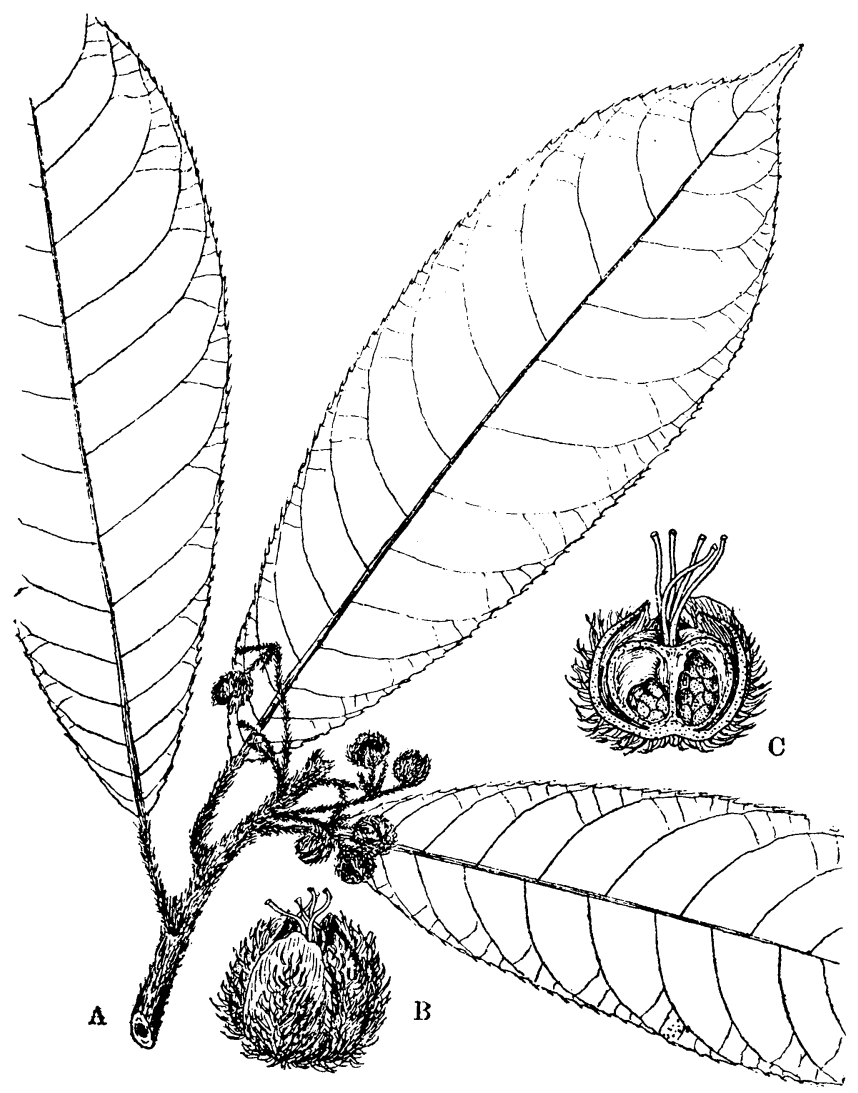

Fig. 2. Saurauia momiensis KAN. et HAT. (No. 14157)

A Branchlet with flowers $\times 2 / 3$. B Young fruit $\times 2$. C The same in $1.5 . \times 21 / 2$.

bracteolis subulatis circ. $3 \mathrm{~mm}$. longis. Sepala ovato-elliptica, apice obtusa, 6-7 mm. longa, dorso dense hirta ; petala obovato-elliptica, circ. $7 \mathrm{~mm}$. longa, olabra; stamina numerosa, antherae circ. $1 \mathrm{~mm}$. longae, filamentis cire. $2 \mathrm{~mm}$. !ongis ; ovarium apice tantum sparse hirtum, styli 5, liberi, circ. $3 \mathrm{~mm}$. longi.

No. 14157 Kanehira-Hatusima, Momi, 60 miles south of Manokwari, April 10, 1940. In edge of rain-forests along the trail to Lake Angi at about $300 \mathrm{~m}$. altitude. 
This is well characterized by its very short inflorescences and its glabrous ovaries with hispid tips. This may be contrasted with Saurauia altissima ZIPPEL which has different leaves with round bases.

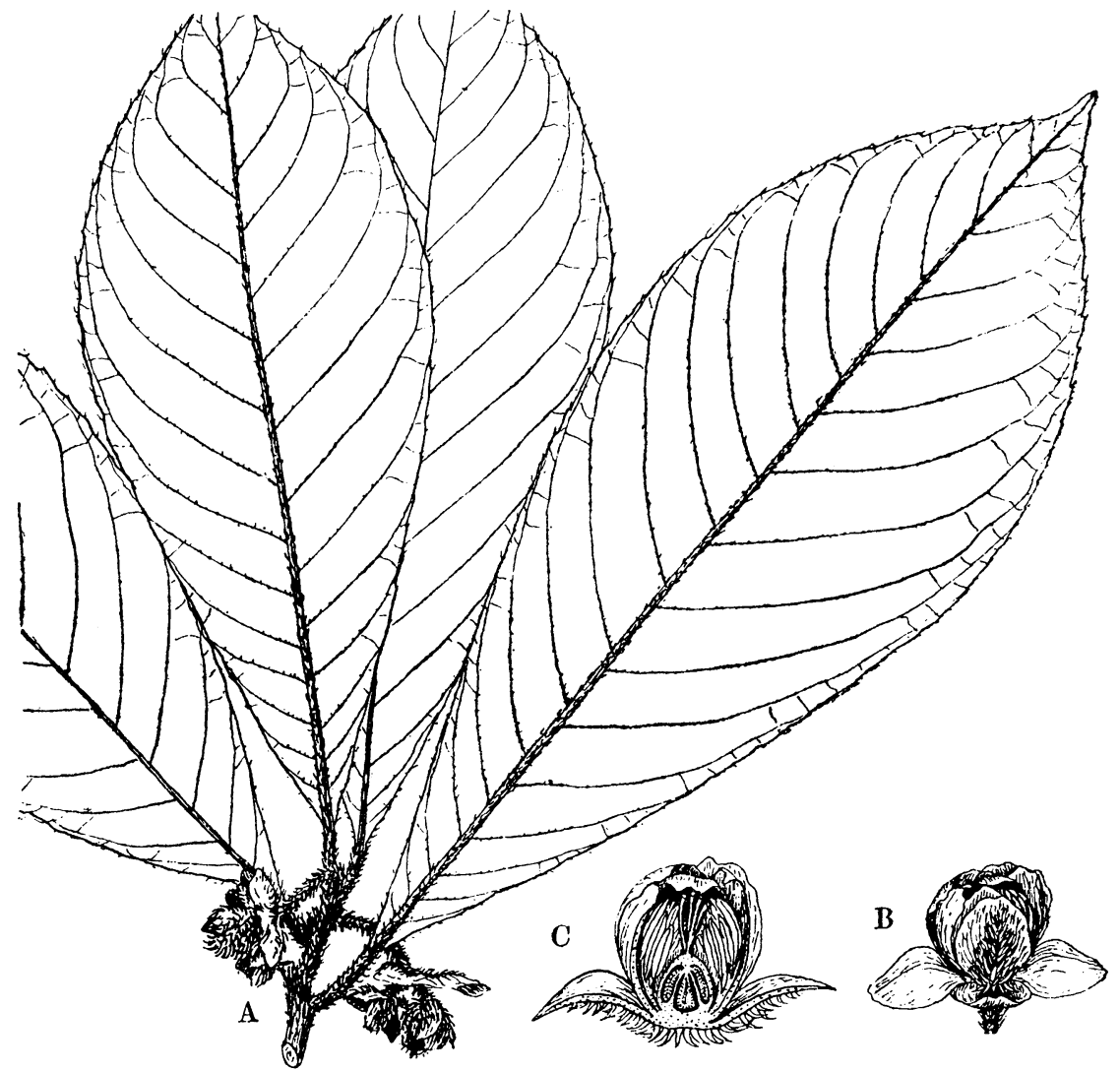

Fig. 3. Saurauia obvallatoides KAN. et ḢA. (No. 11880)

A Branchlet with flowers $\times 2 \%$. B Flower $\times 1 \frac{1}{3}$. C. The same in $1 . s . \times 1 \frac{1}{2}$.

Saurauia (§Setosae) obvallatoides Kanehira et Hatusima sp. nov. Fig.3.

Frutex ad $6 \mathrm{~cm}$. altus, ramuli subteretes paleis pallide fuscescentibus circ. $2 \mathrm{~mm}$. longis dense vestiti, circ. $4-5 \mathrm{~mm}$. crassi. Folia elongato-obovata, papyracea, 16-20 cm. longa, 6-8 cm. lata, apice breviter acuminata, basi sensim angustata, margine remote setuloso-serrulata vel repandata, supra glabra, subtus ad costam nervosque paleis conspersa cetera glabra, nervi laterales circ. 15, adscendentes, petiolo $1.5-2.5 \mathrm{~cm}$. longo. Inflorescentiae axillares dichasiali-triflorae, pedunculatae, pedunculis $1-2 \mathrm{~cm}$. longis, $1.5 \mathrm{~mm}$. crassis ut pedunculi paleis pallide fuscescentibus densiuscule vestitis, pedicelli $1-1.5 \mathrm{~cm}$. longi, $1 \mathrm{~mm}$. crassi, bracteis foliaceis ovato-ellipticis, apice 
rotundatis basi amplectis, circ. $1 \mathrm{~cm}$. longis, dorso ad medium paleaceis, bracteolis ovatis apice obtuse $6-7 \mathrm{~mm}$. longis, dorso praesertim ad medium hispidis. Flores in alabastro circ. $1 \mathrm{~cm}$. diametro; sepala ovato-rotundata, concava, circ. $9 \mathrm{~mm}$. longa, dorso longe denseque hispida margine glabra excepta; petala obovata apice rotundata circ. $8 \mathrm{~mm}$. longa glabra; stamina numerosa, antherae lineari-oblongae apiculatae circ. $3 \mathrm{~m}$. longae; ovarium villosum, styli 5 , liberi, circ. $2 \mathrm{~mm}$. longi.

No. 11880 Kanehira-Hatusima, Chaban, $30 \mathrm{~km}$. inward of Nabire, Feb. 29, 1940. In rain-forests at $300 \mathrm{~m}$. altitude.

This species is to be referred to the series Setosae and represents a transition to the Obvallatae; it seems to be related to Saurauia alkamaarensis LAATr. and Saurauia trachlasia DiELs, but it is easily distinguished from the first by its much larger leaves with more numerous lateral nerves and different inflorescences; from the latter by its somewhat smaller and narrower leaves which are glabrous above.

Saurauia ( $\$$ Setosuc) purpurellofolia Kaneinra et Hatusima sp. nov. Fig. 4.

Frutex ad 2-3 m. altus, rami novelli paleis pallide fuscis circ. $5 \mathrm{~mm}$. loneris molliter vestiti, circ. 5-6 $\mathrm{mm}$. crassi. Folia papyracea, oblanceolata, 20-28 cm. longa, 5.5-7.5 cm. lata, apice acuta, basi sensim angustata, margine subintegra, supra sparse fusco-hirsuta, subtus ad costam nervosque paleis fuscis dense vestita, nervi laterales circ. 14 vel 15 , adscendentes, supra paullo subtus prominente elevati; petiolo $2-2.5 \mathrm{~cm}$. longo ut ramulis paleis fuscescentibus dense vestito. Flores polygami ?, solitarii raro terni, axillares, longe pedunculati, pedunculo $3-4 \mathrm{~cm}$. longo, bracteae inconspicuae; sepala 5 , ovato-elliptica, apice obtusa, extus longissime setosa circ. $1 \mathrm{~cm}$. longa, 5-8 $\mathrm{mm}$. lata; petala anguste obovata circ. $1 \mathrm{~cm}$. longa, $3.5 \mathrm{~mm}$. lata; stamina numerosa, circ. $3 \mathrm{~mm}$. longa; ovarium glabrum depresso-globosum cire. $4 \mathrm{~mm}$. latum, styli 5, liberi, $4 \mathrm{~mm}$. longi.

No. 12489 Kanehira-Hatusima, Patema, Nabire, March 6, 1940; in rain-forests at about $300 \mathrm{~m}$. altitude. No. 11811 Kanehira-Hatusima, Chaban, Nabire, Feb. 28, 1940; in rain-forests at about $300 \mathrm{~m}$. altitude. A shrub, leaves purplish underneath.

This may be referrable to'Saurauia mamberana Diess and Saurauia idenburgensis A. C. SM.

Squamulosue.

?Saurauia monadelpha ScirefF. in Ann. .Jard. Bot. Buit. 1 (1876) 8; Difls in ENGL. 1. c. 447.

No. 13338 Kanehira-Hatusima, Momi, about 60 miles south of Mano- 


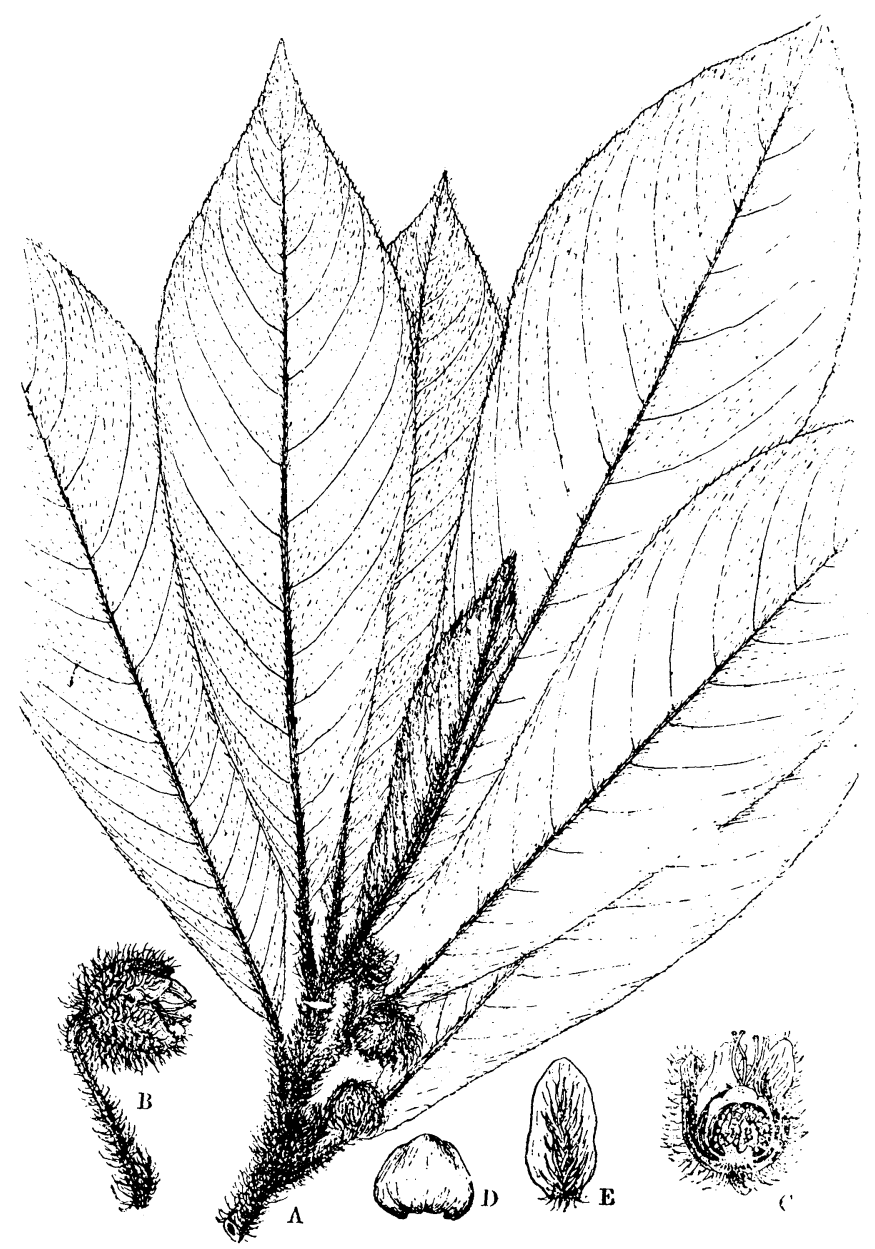

Fig. 4. Saurauia purpurellofolia Kar. et HAT. (No. 11811)

A Branchlet with fruits $\times 1 / 2$. P) Immature fruit $\times 1$. C, The same in $1 . \mathrm{s} . \times 1 \frac{1}{s}$. I) Ovary $\times 1 \frac{1}{1 / 8}$. E Petal $\times 1 \frac{1}{8}$.

kwari. April 3, 1940. In high rain-forests at about $100 \mathrm{~m}$. altitude. A small tree up to $5 \mathrm{~m}$. in height.

Distrib. Endemic, the trpe was from Andai, north-western New Guinea.

Saurauia (\$ Squamulosue) nabirensis Kanemua et Hatesima sp. nov. Fig. 5.

Frutex ad $3 \mathrm{~m}$. altus, ramuli squamulis pallidis vestiti mox glabrati. 3-4 mm. crassi. Folia obovato-oblonga, 9-13 cm. longa, 3.5-4.5 cm. lata, chartacea, apice acute acuminata, basi angustata, margine calloso-serrulata. supra glabrata sparse papillosa, subtus pilis stellatis rufescentibus in costa 
nervosque densiuscule vestita, costa media supra vix elevata, squamis rufis, sparse vestita, subtus bene elevata, infra medium squamis pallidis circ. $1 \mathrm{~mm}$. longis et pilis stellatis conspersa, nervi laterales circ. 10, arcuatim adscendentes, ut nervulis pilis fasciculatis rufis densiuscule vestiti; petiolo 0.8-1.8 cm. longo pallide squpamato. Inflorescentiae axillares longe pedun-

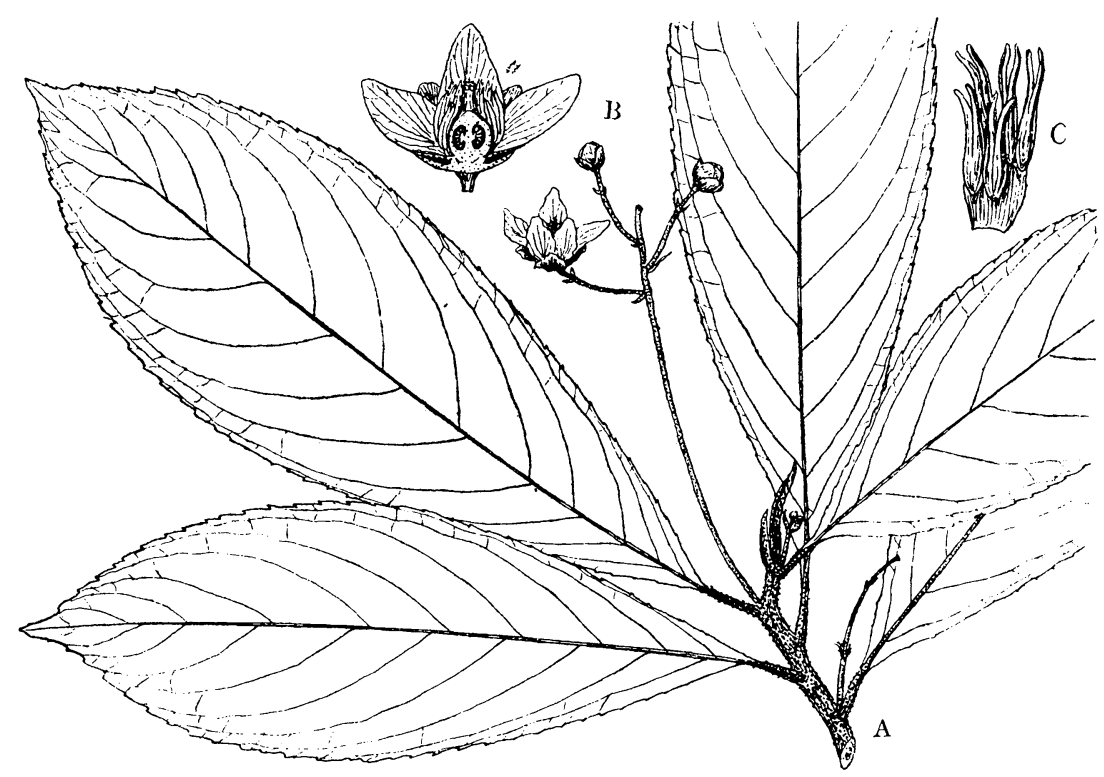

Fig. .). Saurauia nabirensis KAx. et HАT. (No. 12528)

A Branchlet with flowers $\times 2 \%$. B Flower in 1. s. $\times 1 \frac{1 / 3 .}{\text { ( }}$ S Stamens $\times 5$

culatae, pauci florae (4 vel 5) rufo-tomentosae, pedunculis circ. $5 \mathrm{~mm}$. longis $1 \mathrm{~mm}$. crassis, pedicelli $1.5-2 \mathrm{~cm}$. longi bracteolis subulatis $2-6 \mathrm{~mm}$. longis ; sepala majora 3 ovato-orbicularia, apice obtusa, cire. $5 \mathrm{~mm}$. longa. dorso sparse rufo-pubescentia ; petala rosea, anguste ovata, apice obtusa, cire. $8 \mathrm{~mm}$. longa, glabra; stamina numerosa, antherae lineari-oblongae, circ. $2 \mathrm{~mm}$. longae, apice usque ad medium bifilae; ovarium glabrum, stylo 1, eirc. $2 \mathrm{~mm}$. longo, stigmate capitato.

No. 12528 Kaneinra-Hatusima, Semnen, Nabire, March 7, 1940. In rain-forests at about $400 \mathrm{~m}$. altitude.

This is closely related to Saurauia G.jellerupii LaUTB., from which it differs by its smaller and narrower leaves with prominently ascendent lateral nerves and its rufous tomentum of the smaller inflorescences with fewer flowers.

$\S R u f a e$.

Saurauia capitulata A. C. Sмгтн, MSS. Fig. 6. 


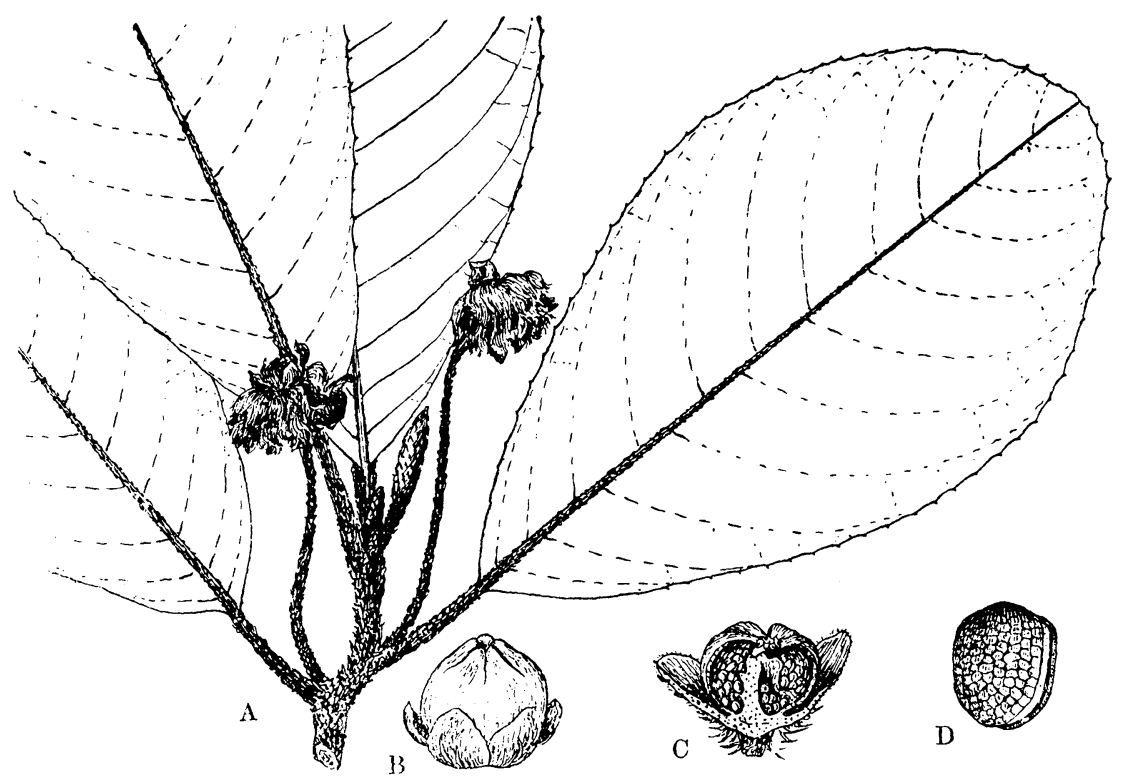

Fig. 6. Saurauia capitulata sмiтн. (No.13886)

A Branchlet with fruits $\times \frac{2 / 3}{2}$ B Fruit $\times 1 \frac{1 / 2}{2}$ C The same in $1 . \mathrm{s} . \times 1 \frac{1}{2}$. D Seed.

Nos. 13886, 13768 Kanehira-Hatusima, Angi, Arfak Mts., April 7-8, 1940. In secondary forests, Lake Giji, Iray at about $1,900 \mathrm{~m}$. altitude. A small tree, 3-5 m. in height, flowers pink, anther brownish.

Distrib. Endemic, the type probably from north-eastern Dutch New Guinea.

\section{\$ovallatae.}

Saurauia Naumannii Diels in Engl. Bot. Jahrb. 57 (1922) 448; var. longipedunculata Kanehira et Hatusima var. nov. Saurauia Naumannii (non Diess) sensu Diels in Nova Guinea 14 (1924) 81.

A typo recedit capitulo circ. duplo majore, pedunculis longioribus ad $10 \mathrm{~cm}$. longis, foliis majoribus, $30-32 \mathrm{~cm}$. longis.

No. 12652 Kanehira-Hatusima, Slieber, Nabire, March 9, 1940. In rain-forests along Boemi River at about $300 \mathrm{~m}$. altitude. A small tree, $4 \mathrm{~m}$. in height, flowers white.

Distrib. Species Bismarck Archipelago, variety endemic.

Saurauia ( Obvallatae) rufinervis Kanehira et Hatusima sp. nov. Fig. 7. Frutex ad $4 \mathrm{~m}$. altus, ramuli petiolique pilis cinereo-rufescentibus 
et paleis stramineis circ. $2-3 \mathrm{~mm}$. longis lanatim vestiti, circ. $5 \mathrm{~mm}$. crassi. Folia obovato-lanceolata ad lanceolata, tenuiter coriaced, $20-25 \mathrm{~cm}$. longa, $6.5-8 \mathrm{~cm}$. lata, apice breviter acuminata, basi acuminato-angustata ad petiolum cire. $2 \mathrm{~cm}$. longum \pm decurrentia, margine setis antrorsum curvatis, circ. 2-3 mm. longis praedita, utrinque primo rufo-tomentella mox supra glabra, subtus araneosa, nervi laterales utrinsecus 14 vel 15 , ut costa nervisque rufescento-tomentosi. Inflorescentiae axillares capitulatae nutantes, $2.5-3 \mathrm{~cm}$. diametro, pedunculis circ. $5 \mathrm{~cm}$. longis paleis stramineis $3-4 \mathrm{~mm}$. longis et pilis cinereo-rufescentibus tomentosim vestitis; bracteae suborbiculares circ. $3 \mathrm{~cm}$. longae, primo a se alte adnatae, dorso ad medium longe paleaceae, cetera glabrae. Flores saepius 2, sessiles, polycamo-dioeci?, bracteolae sepalaque

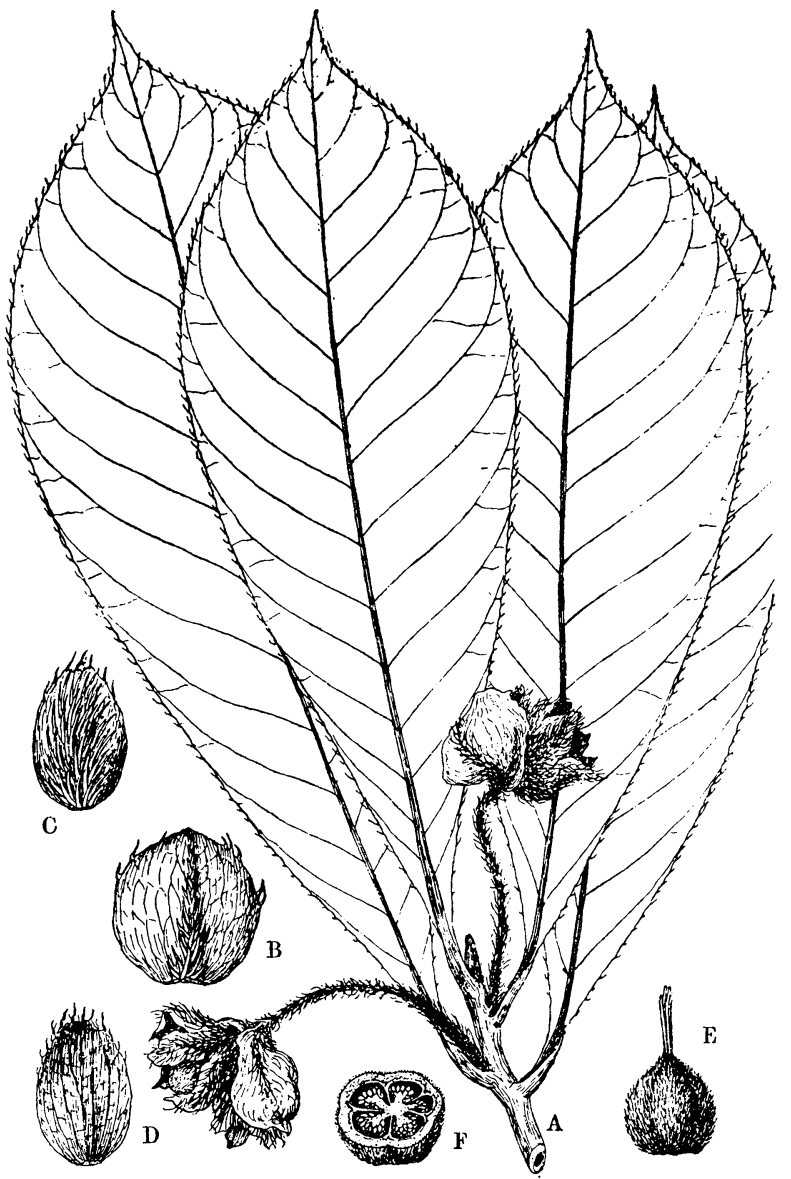

Fig. 7. Saurauia rufinervis KAN. et HAT. (No. 13335)
A Branchlet with flowers $\times 3 / 10$.
B Outer bract $\times 1 / 2$.
C Inner bract (upper surface) $\times 1 / 8$.
D The same (immer surface) $\times 1 \frac{1 / 8}{8}$.
E Pistil $\times 1 \frac{1}{2}$. F Ovary in c. s.

dorso paleis stramineis ad $9 \mathrm{~mm}$. longis basi $1 \mathrm{~mm}$. latis dense praedita, bracteae 2 , late ovatae concavae, circ. $1.5 \mathrm{~cm}$. longae; sepala 5 ovata vel late ovata, $1.2 \mathrm{~cm}$. longa; petala et stamina nulla ?; ovarium subglobosum dense pilosum, styli 5 , liberi, $5 \mathrm{~mm}$. longi.

No. 13335 Kanehira-Hatusima, Momi, 60 miles south of Manokwari, April 3, 1940. In rain-forests at about $100 \mathrm{~m}$. altitude.

This species is to be referred to the series Obvallatae and represents 
a transition to the Rufac; it seems to be comparable with Saurauia novoguineensis ScInefF. and Saurauia decurrens LAUtb. but differs from the both by having shorter petioles and fewer lateral nerves.

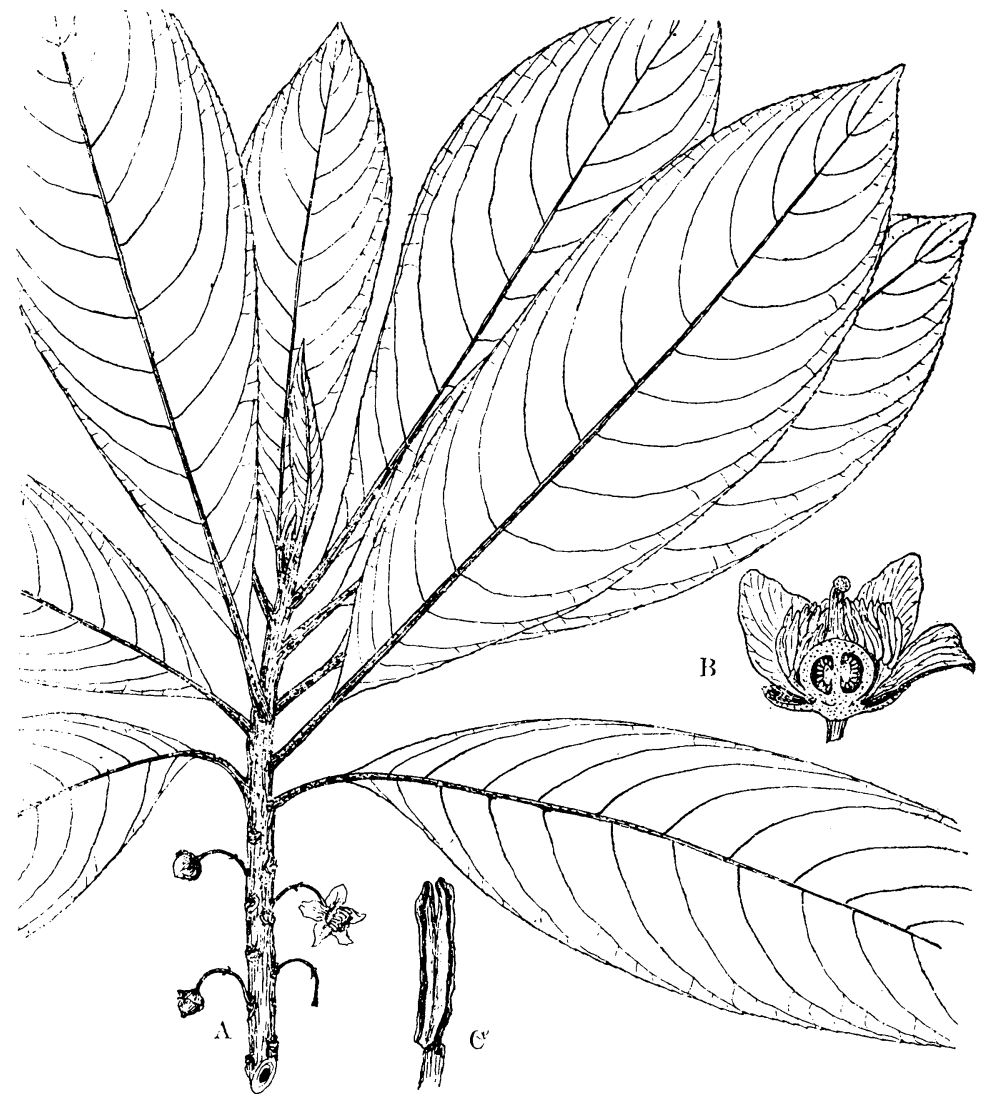

Fig. 8. Saurauia uarenensis Kan. et HAT. (No. 13107)

A Branchlet with flowers $\times \%$ B. B Flower in l. s. $\times 2$. C. stamen.

$\$$ Ramiflorae.

Saurauia ( $\S$ Ramiflorae) warenensis KaneHira et Hatusima sp. nov. Fig. 8.

Arbor ad 15 m. alta, ramuli teretes, novelli squamulis fuscescentibus sparse vestiti, mox glabrescentes (ire. 4-5 mm. (rassi. Folia fasciculata, chartacea, oblonga arl oblonæo-oblanceslata, 12-13 cm. longa, 4-5 cm. lata, apice brevissime acuminata, basi acuta vel obtusiuscula, margine callososerrata, squamulis supra in costa subtus in costa nervosque hinc ince dispersis praedita, ceterum glabra, in sicco supra castanea, subtus pallidiora, nervi laterales 14 vel 15, arcuato-patuli, petiolo circ. $1.5 \mathrm{~cm}$. longo, $2 \mathrm{~mm}$. 
crasso, sparse squanuloso. Flores solitarii, raro bini e ramis defoliatis (rassioribus orti, pedunculi graciles circ. 1.5 cm. longi, $0.8 \mathrm{~mm}$. crassi, fere clabri, ad medium bracteae squamiformes conspersi. Sepala late ovata glabra, circ. $3 \mathrm{~mm}$. longa, $2.5 \mathrm{~mm}$. lata, apice acutiuscula; petala anguste ovata, rosea, apice obtuse acuta, $6-7 \mathrm{~mm}$. longa, 4-5 mm. lata, glabra ; stamina numerosa, circ. $4 \mathrm{~mm}$. longa, antherae oblongae, circ. $2 \mathrm{~mm}$. longae, filamentis adnatis; ovarium subglobosum cire. $3 \mathrm{~mm}$. longum, stylo 1 , apice declinato, circ. $2 \mathrm{~mm}$. longo.

No. 13107 Kanehira-Hatusina, Waren, 60 miles south of Manokwari, March 26, 1940. In rain-forests on a limestone mountain at about $600 \mathrm{~m}$. altitude. A tree with straight bole, up to $15 \mathrm{~m}$. in height and $40 \mathrm{~cm}$. in diameter.

This may be referred to the series Ramiflorae, thought it shows a transition to the Squamulosac; it seems to be related to Saurauia Rodatzii Lautr., which has five liberate styles and? smaller calyxes.

\section{$\$$ Armatue.}

Saurauia (\$limatac) horrida Kanemira et Hatusima sp: nov.

Fig. 9.

Frutex ad $1 \mathrm{~m}$. altus, rami ramulique subteretes, rubescentes, aculeis $3-5 \mathrm{~mm}$. longis, basi $1.5-4 \mathrm{~mm}$. latis dense vestiti. Folia oblonga, chartacea, 11-22 cm. longa,

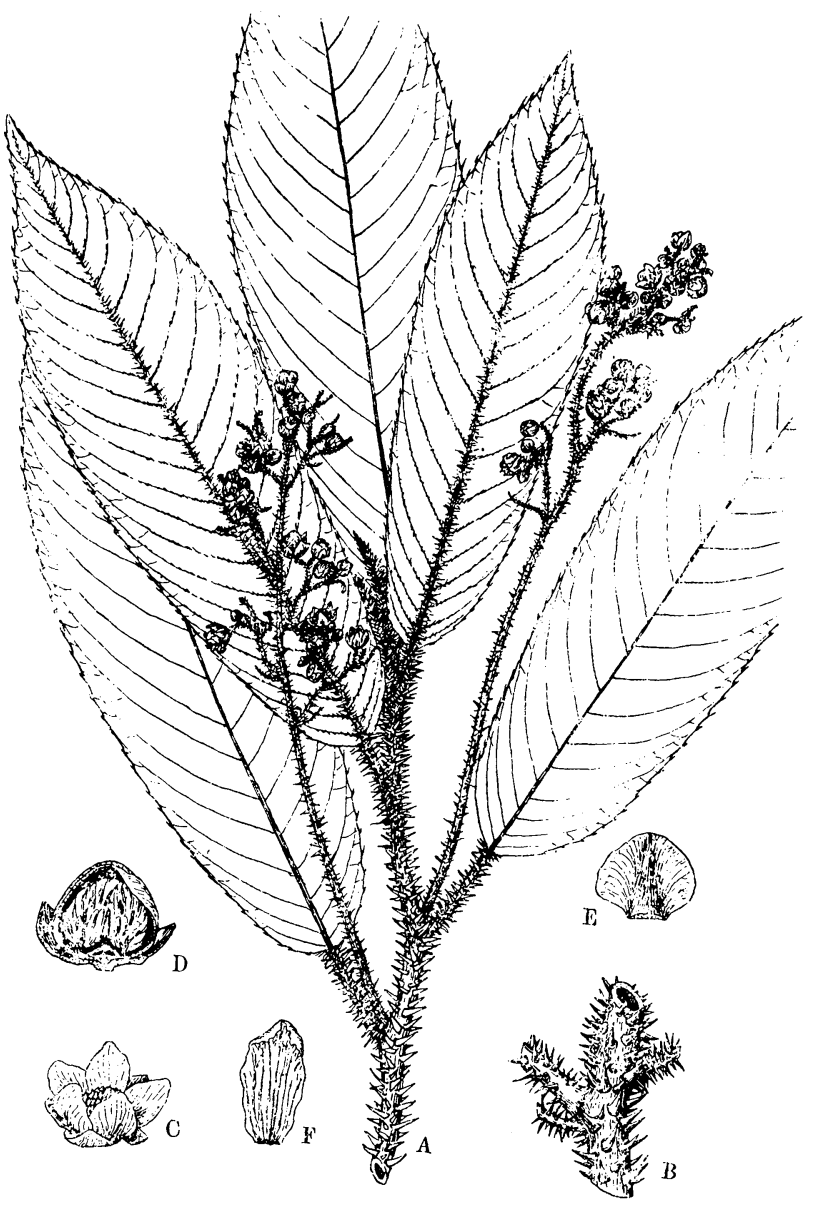

Fig. 9. Saurauia horrida KAN. et HAT. (No. 13552)

A Branchlet with flowers $\times 1 / 2$.

B A portion of branchlet. C Flower $\times 1 \frac{1}{2}$.

D The same in $1 . \mathrm{s} . \times 1 \frac{1}{2}$.

E Sepal $\times 1 \frac{1}{2}$. F Petal $\times 1 \frac{1 / 2}{}$. 
4-6.5 cm. lata, apice breviter acuminata, basi subrotundata, margine spinuloso-serrulata, supra in siceo brunnea, hirtis eystolithiformibus densiuscule notata, subtus setulosa, costa media impressa, subtus prominente elevata, aculeis et setis rigidis densiuscule conspersa. Inflorescentiae paniculatae axillares ad $15 \mathrm{~cm}$. longae, aculeatae, pedunculis ad $8 \mathrm{~cm}$. longis, bracteolis linearibus $3-10 \mathrm{~mm}$. longis extus setulosis, pedicelli $3-10 \mathrm{~mm}$. longi setulosi. Flores polygamo-dioeci ? (masculi tantum visi); sepala ovata, acutiuscula circ. $4 \mathrm{~mm}$. longa dorso setulosa; petala ovato-elliptica circ. 8 mm. Ionga, dorso glabra; stamina numerosa, antherae circ. $2.5 \mathrm{~mm}$. longae, ovarii rudimentum nullum.

No. 13552 Kanehira-Hatusina, Angi Arfak Mts., April 5, 1940. On edge of mossy forests, Lake Gita at about 1,900 m. altitude.

This is well characterized by its paniculate inflorescences with polygamo-dioeceous flowers. The nearest alliance of this species may be with Saurania aculeata LautB., from which it differs by its much robust spines of the branchlets and petioles, its larger leaves with more numerous lateral nerves, and its large panicles with polygamo-dineceous flower's.

\section{R. Kanehira and S. Hatusima: Sapindaceae.}

Allophylus timorensis (DC.) BL. Rumph. 3 (1847) 130; RadLK. in ENGL. Bot. Jahrb. 56 (1920) 306 et ENGL. Pflanzenr. 98 (1934) 587.

No. 11799 Kanehira-Hatusima, Papaya, Nabire, Feb. 27, 1940 ; in secondary forests at about $100 \mathrm{~m}$. altitude. No. 12888 Kanemira-Hatusmin, Nabire, March 15, 1940; in edge of high rain-forests at $3 \mathrm{~m}$. altitude.

Distrit. Formosa to the Nicobar Islands. though Malaya to Samoa and New Caledonia.

\section{Cupaniopsis ( $\$$ Elattopelatum) longifoliolata Kanemira et Hatusima} sp. nov. Fig. 10.

Arbuscula 4-5 m. alta, rami teretes circ. $1 \mathrm{~cm}$. crassi petiolique pallide rufo-villosuli. Folia abrupte pinnata, longe petiolata, petiolo $12-22 \mathrm{~cm}$. longo circ. $4 \mathrm{~mm}$. crasso, rhachis 19-34 cm. longa. Foliola subopposita, papyracea, subsessila, plerumque 15-17, anguste oblonga vel oblonga, 13-23 cm. longa (maxima $27 \mathrm{~cm}$. longa) circ. $5 \mathrm{~cm}$. lata, apice breviter acuminata basi oblique cuneato-subrotundata, margine serrato-dentata, supra in sicco fusco-viridia glabra, subtus ad nervos venasque dense pilosa, nervis lateralibus utrinsecus 15-27, venulis reticulatis subtus prominentibus. Paniculae supraaxillares, masculae amplae ad $15 \mathrm{~cm}$. longae et latae; ramis elongatis thyrsoideis apice flores singulos pedicellatos gerentibus, pedicellis $2.5-3 \mathrm{~mm}$. longis, bracteae bracteolaeque subulatae ut sepala villosulae. Sepala latiuscule imbricata, 
interiora obovato-elliptica $2.5 \mathrm{~mm}$. longa, $1.5 \mathrm{~mm}$. lata, basi unguiculata dorso toto intus basi tantum sericeo-pilosa. Petala sepalis angustiona brevioraque oblongo-elliptica, apice obtusiuscula, circ. $1 \mathrm{~mm}$. longa, $0.5 \mathrm{~mm}$. lata, dorso basin versus pilosa, intus glabra, squamulis 2 villosulis. Discus

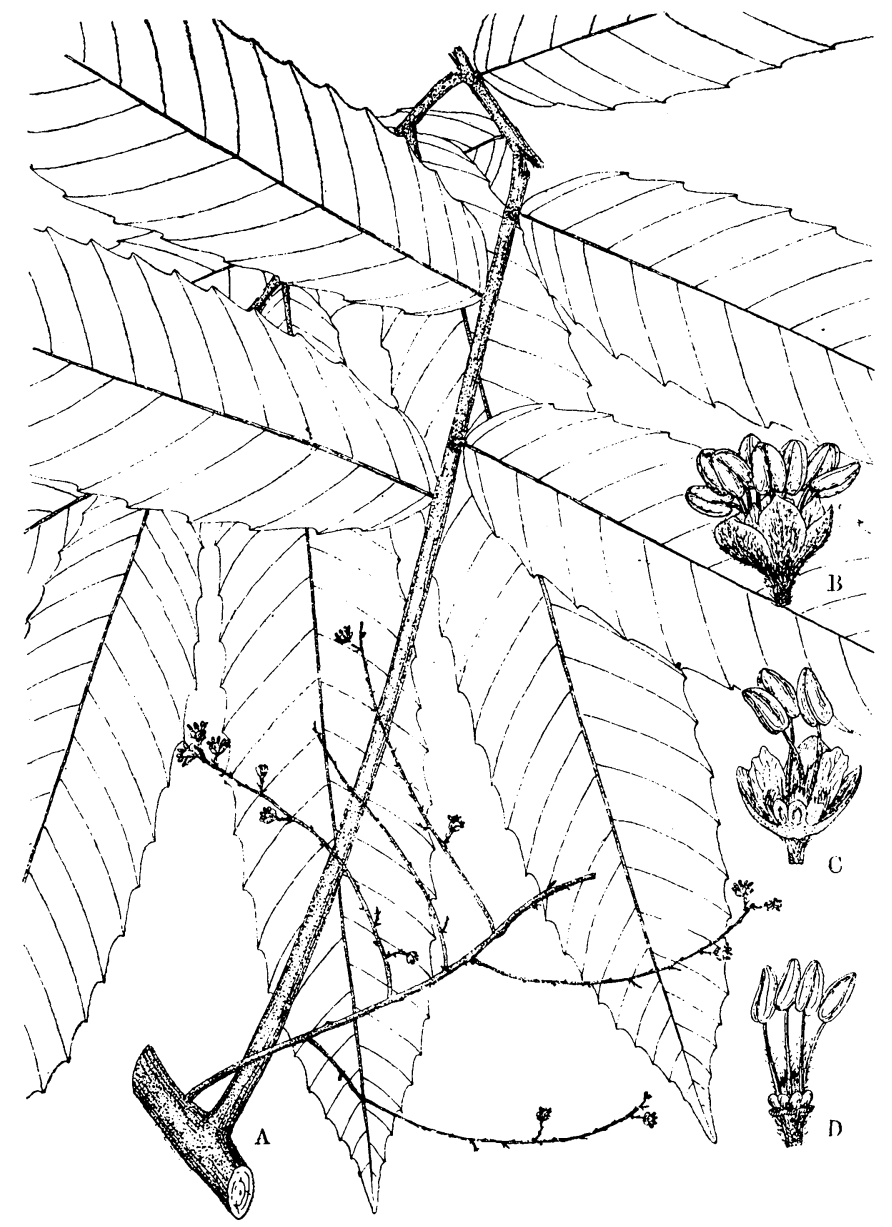

Fig. 10. Cupaniopsis longifoliolata KAN. et HAT. (No. 14135)

A Branchlet with staminate flowers $\times 1 / 2$. B Staminate flower $\times 3 \frac{3}{4}$.

C The same in $1 . \mathrm{s} . \times 3 \frac{3}{4}$. D Stamens and disc. $\times 3 \frac{34}{4}$.

glaber. Stamina 8 , filamentis villosulis circ. $2 \mathrm{~mm}$. longis, antherae ovatooblongae circ. $1.5 \mathrm{~mm}$. longae; ovarii rudimentum nullum. Capsula rubra subglobosa, subsessilia, circ. $1 \mathrm{~cm}$. lata, extus tomentella.

No. 12940 (fr. type) Kanehira-Hatusima, Waren, 60 miles south of Manokwari, March 21, 1940; in rain-forests at about $200 \mathrm{~m}$. altitude. No. 
14135 (fl. type) Kanlenra-Hatesima, Momi, April 11, 1940; in high rainforests at $100 \mathrm{~m}$. altitude.

This may be ontrasted with Cupceniopsis curvidens Raduk. from which it differs chiefly by its much fewer and larger leaflets and its larger flowers.

Dodonaea viscosa (LINn.) J ACQ. var. vulgaris Ben'Th. Fl. Austr. 1 (1836) 476 ; Radlk. in Engl. Pflanzenr. 98 (1934) 1368.

Nos. 13639, 13900 Kanehrra-Hatusima, Angi, Arfak Mts., April 8. 1940. Abundant in secondary forests on an inundation area, Lake Giji, Iray at about $1900 \mathrm{~m}$. altitude.

Distrib. A common strand plant in Tropies, but rare at high altitude.

Elattostachys Zippeliana (BL.) RAduk. Sap. Holl.-Ind. (1877) 12, 43 (82, 113) et ENgL. Pflanzenr. l. c. 1264.

No. 12665 Kanemira-Hatusima, Slieber, Nabire, Narch 9, 1940. In edge of fringing rain-forests at $300 \mathrm{~m}$. altitude. A tree up to $15 \mathrm{~m}$. in height.

Distrib. Endemic.

Erioglossum rubiginosum (Roxв.) BL. Rumph. 3 (1847) 118 in obs;

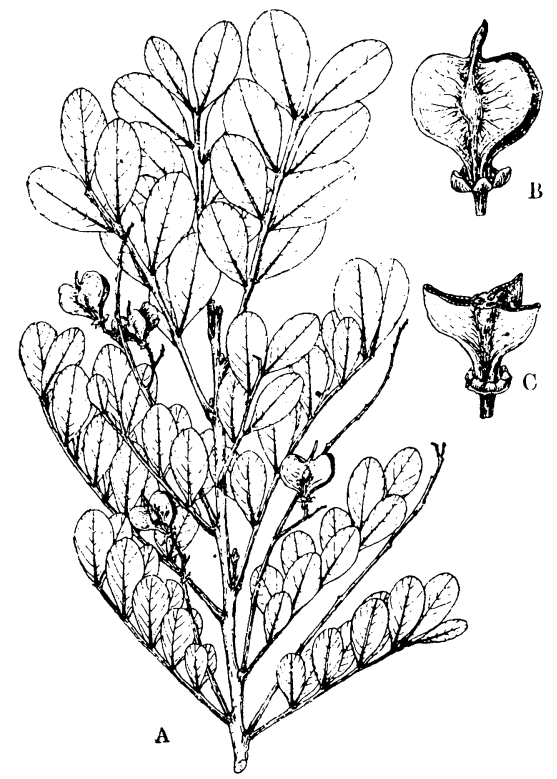

Fig. 11. Guioa amabilis KAN. et HAT. (No. 13147)

A Branchlet with flowers $\times \% / 3$.

I3 Fruit $\times 1 \frac{1 / 3}{\text {. }}$

(:) The same in c.s. $\times 1 \frac{1 / 3}{3}$. RadLK. in ENGL. Bot. l. c. 266 et ENGL. Pflanzenr. 1. e. 493.

No. 13274 Kanehira-Hatusima, Momi, March 30, 1940. In rain-forests at $30 \mathrm{~m}$. altitude. A shrub about $2 \mathrm{~m}$. in height, rare.

Distrib. From India to Cochin-China. through Malaya to north-eastern Australia.

Guioa ( $\$$ Euguioa) amabilis KanEhira et Hatusima sp. nov. Fig. 11.

Frutex ad $3 \mathrm{~m}$. altus, rami nigrescentes, teretes, ramuli juniores dense fuscescento-puberuli circ. 1-1.5 mm. erassi. Folia abrupte pinnata, 4-5 cm. longa; foliola plerumque 4- vel 5-juga, subopposita, coriacea, ovatoelliptica, apice rotundata vel paullo emarginata, margine ad prope apicem tantum uni-crenata cetera integra, 
valde revoluta, basi inaequilatera ad petiolulum perbrevem cuneatim angustata, $1.5-2 \mathrm{~cm}$. longa 8-11 mm. lata, in siceo supra brumnea nitida, subtus glaucina dense acipresseque hirsuta, nervis lateralibus 4-6, supra vix subtus tenuiter elevatis, rhachis foliorum insignius alata ut petiolo cire. $1.5 \mathrm{~cm}$. longo $1 \mathrm{~mm}$. crasso fuscescento-puberula. Infructescentiae axillares paniculatae foliis subaequantes, dense puberulae, ramis thyrsoideis cincinnos breviter stipitatos paucifloros gerentibus. Sepala (sub fructu) suborbicularia, margine ciliata, utrinque glabra, 1.5-2 mm. longa. Discus annularis, sublobatus, glaber. Capsula late obcordata, 3-alato-lobata in stipem brevem 3-angularem attenuata, glabra, apice stylo eirc. $2.5 \mathrm{~mm}$. longo coronata, 8-9 mm. longa, 1-1.2 cm. lata, pedicellis (fructigeris) cire. $1 \mathrm{~mm}$. longis dense puberulis.

No. 13999 Kanehira-Hatusina, Angi, Arfak Mts., April 9, 1940. In open spinneys on the summit of Mt. Koebre at about 2300 m. altitude.

This is closely related to Guioa venusto RaDLK. but differs by its fewer leaflets which are glabrous on both surfaces.

Guioa membranifolia Raduk. in Sap. Holl.-Ind. (1877) 11, 40, 90 et ENGL. Bot. l. c. 282 et ENGL. Pflanzenr. l. c. 1174.

No. 12658 Kanehira-Hatusima, Slieber, Nabire, March 9, 1940. In edge of fringing rain-forests at about $300 \mathrm{~m}$. altitude. A tree about $10 \mathrm{~m}$. in height, fruits red.

Distrib. Bismarck Archipelago and north-eastern New Guinea.

Harpullia cauliflora K. Schum. et Lautb. Fl. Deutsch. Schutzg. Süd. (1901) 424 ; RadLk. in Nova Guinea 8 (1912) 618 et EnģL. Bot. l. c. 312 et Engl. Pflanzenr. 1. c. 1440.

No. 12633 Kanehira-Hatusima, Ayerjat, Nabire, March 9, 1940 ; in edge of fringing rain-forests at $300 \mathrm{~m}$. altitude. No. 12388 KaneniraHatusima, Sennen, Nabire, March 6, 1940; in rain-forests at $300 \mathrm{~m}$. altitude. A shrub, 3-4 m. in height, fruits red.

Distrib. North-eastern New Guinea.

Harpullia ef. fruticosa BL. Rumph. 3 (1847) 179 ; RAdLK. in ENGL. Bot. 1. c. 313 et Pflanzenr. 1. c. 1443.

No. 12896 Kanehira et Hatusima, Nabire, March 16, 1940. In high rain-forests at $3 \mathrm{~m}$. altitude. A shrub $3 \mathrm{~m}$. in height.

Distrib. Endemic.

Harpullia ef. hirsuta RADLk. in Nova Guinea 8 (1912) 618 et ENGL. Bot. 1. c. 315 et Pflanzenr. 1. c. 1450 .

No. 12597 Kanehira-Hatusima, Sennen, Nabire, March 8, 1940; in rain- 
forests at $300 \mathrm{~m}$. altitude. Nos. 11714, 11704 Kanehira-Hatusima, PapayaNabire, Feb. 27, 1940; in rain-forests at about $100 \mathrm{~m}$. altitude. A shrub 2-3 m: in height.

Distrib. North-eastern New Guinea.

Harpullia longithyrsifera Kanehira et Hatusima, sp. nov. Fig. 12.

Arbor parva ad 4-5 m. alta, ramuli juniores teretes pilis stellatis fuscis sparse vestiti mox glabri. circ. $4 \mathrm{~mm}$. crassi. Folia abrupte pinnata, 5- vel 6-juga, subopposita, petiolo tereti $4-5 \mathrm{~cm}$. longo, rhachi tereti $16-21 \mathrm{~cm}$. longa ; foliola chartacea, ovato-oblonga $10-18 \mathrm{~cm}$. longa $4.5-$ $6.5 \mathrm{~cm}$. lata, inferiora minora ovata vel anguste ovata, apice obtuse breviterque acuminata, basi acuta subaequilatera, margine integra, petiolulo 3-5 mm. longo, utraque facie prominule reticulato-venosa, glabra, nitidula, in sicco flavo-viridia. Inflorescentiae ad apicem ramorum axillares 20 $48 \mathrm{~cm}$. longae, stellatopuberulae, fere a basi polychasia subsessilia

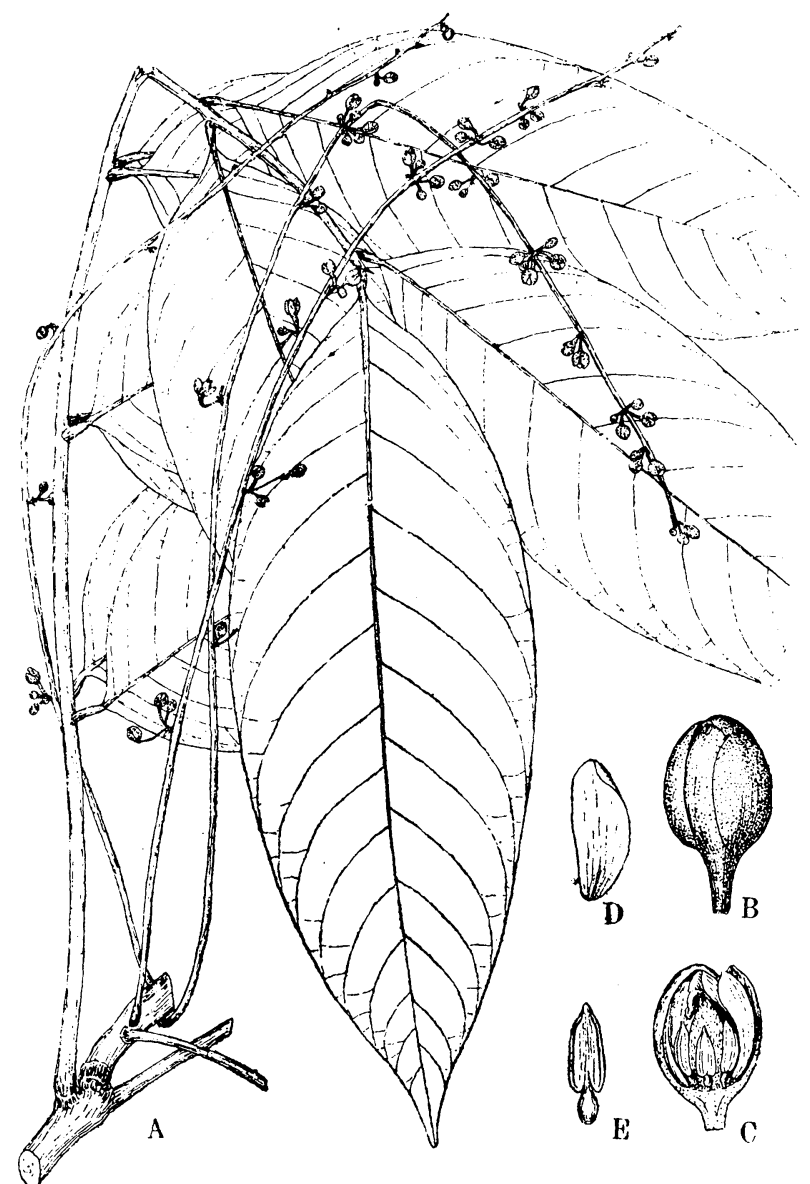

Fig. 12. Harpullia longithyrsifera KAN. et HAT. (No. 14234)

A Branchlet with flowers $\times 1 / 2$. B Flower bud $\times 3$.

C The same in 1. s. $\times 3$. D Petal $\times 3$. E Stamen $\times 3 / 4$. brevia ramosa pluriflora remotiuscula gerentes, pedicelli $3-7 \mathrm{~mm}$. longi ut sepala brevissime fuscescento-tomentosi. Sepala subelliptica circ. $5 \mathrm{~mm}$. longa, $3 \mathrm{~mm}$. lata. Petala carnosula ovato-oblonga vel suboblonga circ. 3 $\mathrm{mm}$. longa, $2 \mathrm{~mm}$. lata, glabra. Stamina 5 , circ. $3 \mathrm{~mm}$. longa, antherae circ. 
$2 \mathrm{~mm}$. longae. Discus pubescens, ovarium tomentellum in stylum circ. 2 mm. longum subulatum attenuatum. Fructus ignotus.

No. 14234 Kanehira-Hatusima, Momi, April 19, 1940. In rain-forests at $3 \mathrm{~m}$. altitude. A myrmecophilous plant.

This may be contrasted with Harpullia reticulata RADLK. which has rufo-tomentellous branchlets.

Harpullia Peekeliana Melchior in Notizbl. Bot. Gart. u. Mus. BerlinDahlem 10 (1928) 279 ; Radlk. in Engl. Pflanzenr. l. c. 1461.

No. 13285 Kanehira-Hatusima, Momi, March 30, 1940. In rainforests at $50 \mathrm{~m}$. altitude. A small tree about $10 \mathrm{~m}$. in height.

Distrib. Hitherto known only from Bismarck Archipelago.

Mischocarpus paradoxus RADLK. in Sitzungsib. Bayer. Akad. 20 (1890) 268 adnol. et Engl. Bot. l. c. 306 et Engl. Pflanzenr. 1. c. 1310.

No. 12825 Kanehira-Hatusima, Bivak Prao, Nabire, March 1\&, 1940. In edge of fringing rain-forests at $100 \mathrm{~m}$. altitude.

I) istrib. South-eastern New Guinea.

Mischocarpus Guillauminiana Kanehira from 'Truk, Caroline sslands does not seem to be distinct from this species.

Mischocarpus sundaicus BL. Bijdr. (1825) 238 ; RADLK. in ENGL. Bot. l. c. 303 et ENGL. Pflanzenr. l. c. 1299.

No. 12516 Kanehira-Hatusima, Sennen, Nabire, March 7, 194v. In edge of fringing rain-forests at $300 \mathrm{~m}$. altitude. A tree $6 \mathrm{~m}$. in height. Distrib. Cochin-China, southern China, Malaya to Philippines and northern Australia.

Pometia pinnata J. et G. Forster Char. Gell. (1776) 110, t. 55, excl. syn.; Radlk. in Nova Guinea 8 (1912) 617 et EngL. Bot. I. c. 271 et EngL. Pflanzenr. l. c. 929 .

No. 11504 Kanehira-Hatusima, Nabire, Feb. 24, 1940. In high rainforests at $2 \mathrm{~m}$. altitude.

Distrib. From Formosa through Malaya to Polynesia.

Rhysotoechia longipaniculata Kanehira et Hatusima nov. sp. Fig. 13.

Frutex $2 \mathrm{~m}$. altus, rami teretes nigro-cinerascentes vel brunneo-cinerascentes, 3-5 mm. crassi, lenticellis magnis conspersi. Medulla ampla. Folia abrupte pinnata, petiolo $1.5-4.5 \mathrm{~cm}$. longo $3 \mathrm{~mm}$. crasso, lenticellato. Foliola obovato-oblonga vel oblongo-elliptica vel oblonga, coriacea, 19-27 cm. longa 6.5-10 cm. lata, apice breviter acuminata, basi acute acuminata, margine integra, in sicco utrinque viridia, nitidula, eglandulosa glaberrima, nervis lateralibus 9 vel 10, arcuatim adscendentibus ut venulis reticulatis utrinque 
prominente elevatis, petioluli valde incrassati rugosi circ. $1 \mathrm{~cm}$. longi $3-4$ $\mathrm{mm}$. crassi. Paniculae $(\delta)$ axillares ramosae laxiflorae, foliis longiores ad $37 \mathrm{~cm}$. longae, sub lente fusco-puberulae, pedunculis $8-14 \mathrm{~cm}$. longis $1.5 \mathrm{~mm}$. crassis, pedicelli circ. $2 \mathrm{~mm}$. longi. Sepala 5 , petaloidea, elliptica vel late

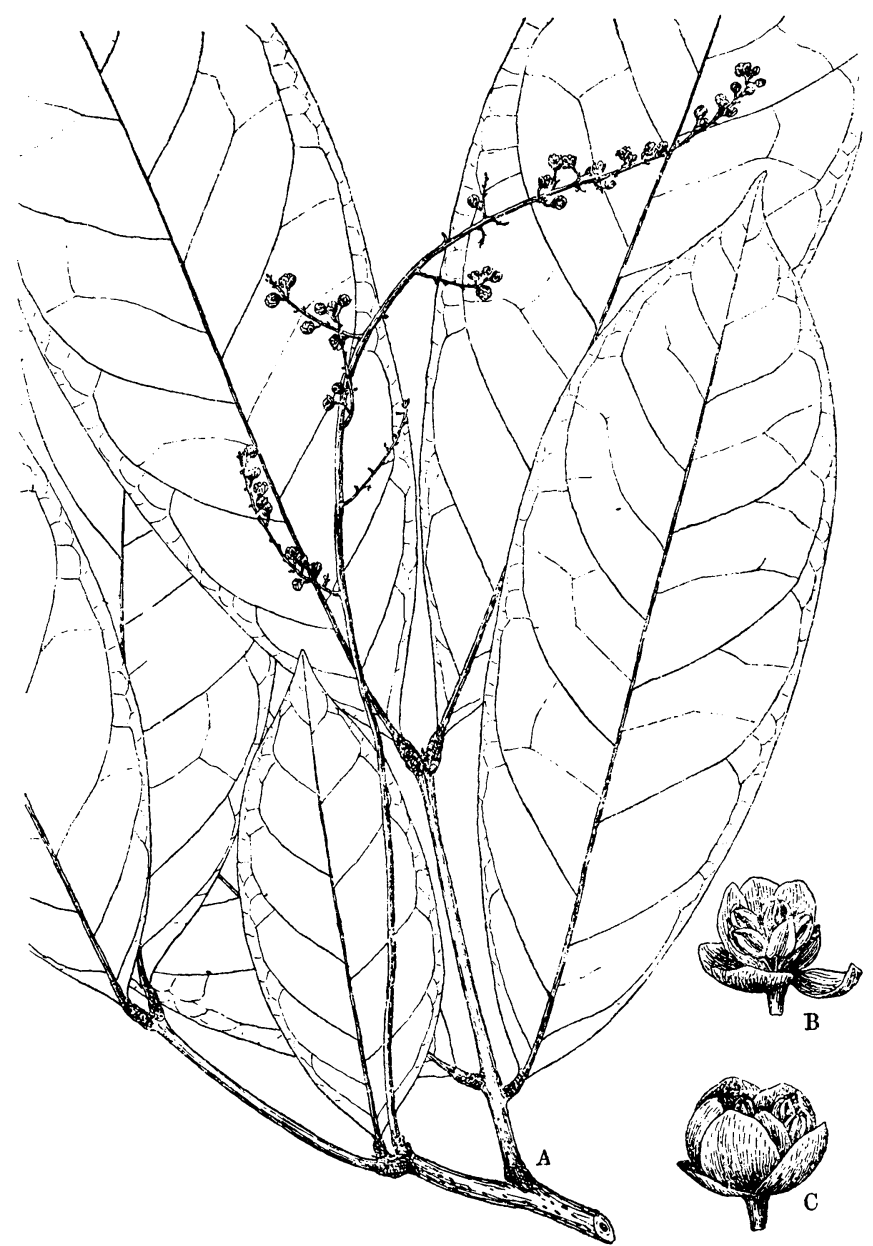

Fig. 13. Rhysotoechia longipaniculata KAN. et HAT. (No. 11534)

A Branchlet with fruits $\times 1 / 2$. B, C Staminate flowers $\times 3$.

elliptica, circ. $3 \mathrm{~mm}$. longa, glabra, margine ciliolata. Petala ovato-elliptica, circ. $3.5 \mathrm{~mm}$. longa, glabra, basi tantum pilosa, margine ciliata. Stamina $\overline{5}$ antherae ellipticae $1 \mathrm{~mm}$. longae, filamentis infra medium incrassatis dense pilosis $2.5 \mathrm{~mm}$. longis. Ovarii rudimentum clavatum glabrum, eire. $0.5 \mathrm{~mm}$. longum. Discus obscurus.

No. 11534 Kanehira-Hatusima, Nabire, Feb. 25, 1940. In edge of 
high diluvial rain-forests at $1 \mathrm{~m}$. altitude.

This is well characterized by its long panicles and large leaflets.

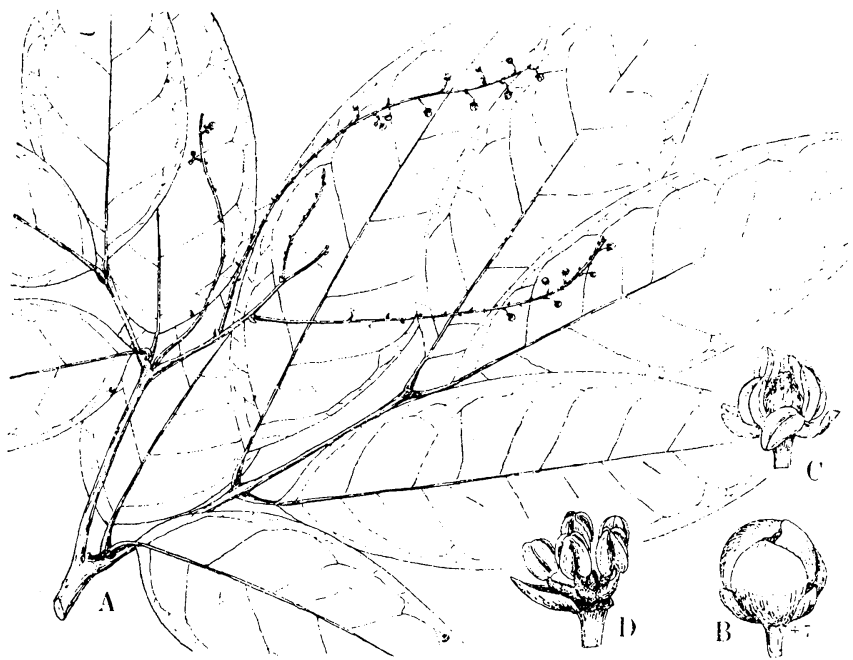

Fig. 14. Rhysotoechia momiensis KAN. et HAT. (No.14148)

A Branchlet with flowers $\times \%$. B Flower bud $\times 5$. Perfect flower $\times 5$. 1) Staminate flower, petals taken off $\times 6$.

Rhysotoechia momiensis Kanehira et Hatusima sp. nov. Fig. 14.

Frutex glaber circ. $1.5 \mathrm{~m}$. altus, ramuli novelli fusco-cinerascentes, vetustiores cinereo-nigrescentes, dense minuteque lenticellati, 2-3 mm. crassi. Medula ampla. Folia abrupte pinnata eum petioli 6-14 $\mathrm{cm}$. longa, petiolo 1-4 cm. longo $2 \mathrm{~mm}$. erasso basi incrassato. Foliola plerumque 6, subopposita, tenuiter coriacea, oblonga vel oblongo-elliptica, 7-15 cm. longa, $2.5-5 \mathrm{~cm}$. lata, apice acutiuscula, basi subrotundata vel acutiuscula ad petiolulum 5-7 mm. longum $1 \mathrm{~mm}$. crassum basi valde incrassatum contracta, margine integra, in sicco supra olivaceo-viridia, subtus virideo-fuscescentia, glabra, haud glandulosa, utrinque prominente reticulato-venosa, nervis lateralibus utrinsecus 9 vel 10, subtus prominetibus. Inflorescentiae in ramis vetustioribus 2-5-ni fasciculatae rarius simplices, paniculatae, polygamo-dioecae ?, pauciflorae, glabriusculae foliis longiores, ad $5-17 \mathrm{~cm}$. longae, ramis elongatis, bracteae bracteolaeque minutae puberulae, pedicelli 1-2.5 mm. longi glabriusculi. Sepala 4 vel 5, petaloidea, 2 exteriora minora ovato-elliptica circ. $2 \mathrm{~mm}$. longa, 2 vel 3 interiora majora late elliptica cire. $3 \mathrm{~mm}$. longa glabra. Petala elliptica vel late elliptica circ. $3 \mathrm{~mm}$. longa, utrinque glabra, basi squamulata, squamulis pilosis. Stamina 5, antherae late ellipticae, $1.5 \mathrm{~mm}$. longae, filamentis $1 \mathrm{~mm}$. longis basi incrassatis et pilosis. Ovarium ovoideum, pilosum. Ovarii rudimentum in floribus $\hat{o}$ subnullum. Discus obscurus. 
No. 14148 Kaneinira-Hatusima, Momi, April 11, 1940. In rain-forests at $100 \mathrm{~m}$. altitude.

This is most closely related to Rhysotoechia ramiflora RADLK. from Celebes which chiefly differs by its larger and broader leaflets.

Sarcopteryx coriacea RadLk. Sapind. Holl. Ind. (1878) 98 (249) et ENGL. Bot. 1. c. 296 et Engl. Pflanzenr. 1. c. 1235.

Nos. 13713 (fr.), 13651 (fl.), 14037 (fr.) Kanehira-Hatusima, Angi, Arfak Mts., April 6-9, 1940. On the open summit of Mt. Koebre at about 2000-2300 m. altitude. A shrub $3 \mathrm{~m}$. in height.

Distrib. Waigiu Island.

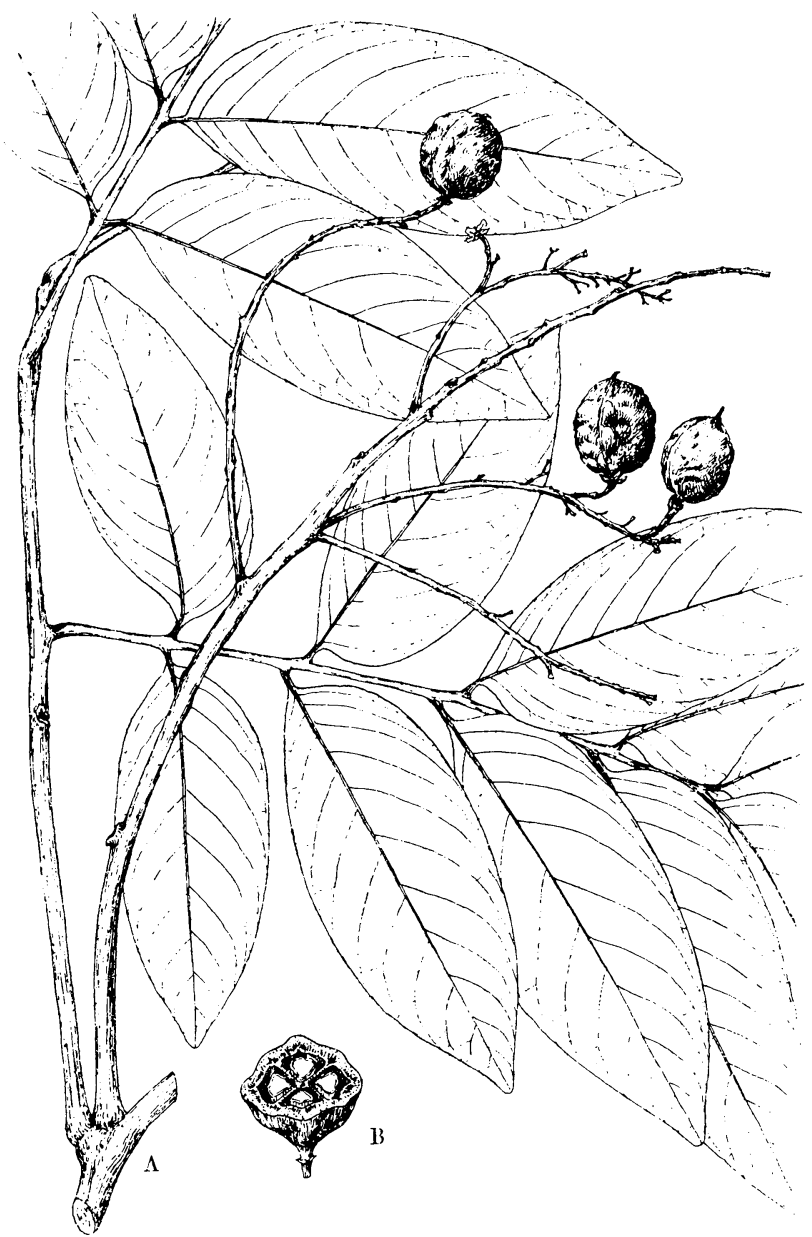

Fig. 15. Tristylopsis novo-guineensis KAN. et HAT. (No. 14249)

A Branchlet with fruits $\times 1 / 2$. B Fruit in c. s. $\times 3 / 4$. 
Toechima hirsutum Radlk. in K. Schum et Hollrung, Fl. Kaiser. Wilhelmsl. (1889) 67 et ENGL. Bot. 1. c. 298 et EngL. Pflanzenr. 1. c. 1253.

No. 12835 Kanehira-Hatusima, Bivak Prao, Nabire, March 12, 1940. In fringing rain-forests at $100 \mathrm{~m}$. altitude. A small tree, $10 \mathrm{~m}$. in height, fruits red.

Distrib. Endemic.

Toechima subteres RadLk. Sapind. Holl. Ind. (1877), 19, 60 (89, 130) et Engl. Bot. 1. e. 298 et ENGL. Pflanzenr. l. e. 1254.

No. 12987 Kanehira et Hatusima, Waren March 21, 7940. In open thickets on a hill at about $300 \mathrm{~m}$. altitude. A small tree $100 \mathrm{~m}$. in height, fruits red.

Tristylopsis * novo-guineensis Kanehira et Hatusima sp. nov. Fig. 15.

Arbor ad $30 \mathrm{~m}$. alta, rami fusci sub lente fusco-pilosi et bene lenticellati, circ. $7 \mathrm{~mm}$. crassi. Folia bipinnata ad $40 \mathrm{~cm}$. longa, petiolo tereti circ. $5 \mathrm{~mm}$. (rasso fusco-piloso. Foliola oblongo-lanceolata \pm falcata ad $13 \mathrm{~cm}$. longa 2.3-4.5 cm. lata, apice obtusa, basi oblique subrotundata, utrinque glabra, supra nitidula, subtus opaca, chartaceo-coriacea. Infructescentiae amplae ad $40 \mathrm{~cm}$. longae, rhachi dense brevissimeque fusco-hirsutae. Fructus subglobosus vel ellipsoideus basi in stipitem circ. $2 \mathrm{~mm}$. longum contractus, leviter 4- (rarius 3-5-) angulatus, apice stylo circ. $1.5 \mathrm{~mm}$. longo coronatus, supra flavido-sriceus, circ. $2.2 \mathrm{~cm}$. longus $1.8-2.2 \mathrm{~cm}$. latus, pedicellis (fructigeris) $5-7 \mathrm{~mm}$. longis $1.2 \mathrm{~mm}$. crassis dense pilosis. Sepala (sub fructu) ovato-elliptica circ. $4 \mathrm{~mm}$. longa $2 \mathrm{~mm}$. lata.

No. 14249 Kanehira-Hatusima, Waren, April 19, 1940. In high rainforests at $3 \mathrm{~m}$. altitude.

This is very closely related to Tristylopsis subfalcata RADLK. from the Philippines, from which it differs chiefly by its somewhat broader and usually 4-gonous fruits.

\section{金平・初島探集ニューギニヤ植物研究 XVII.}

\section{Dilleniaceae 及 Actinidiaceae}

$$
\text { 金本桄三・初島住彥 }
$$

今间我々ガ探集シタ Dilleniaceae 八次ノ 3 屬 3 種デ總テ既知種デアル。

Dillenia philippinensis var. pubiflora MrRn. 完全ナ花ヨ探集スルコトガ出來ナカ

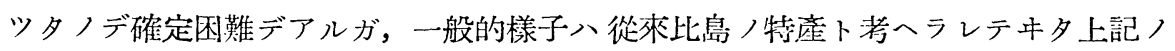
種卜買ナラヌ樣デアル。大キナ葉卜赤色ノ樹玑 ヨ有スル大售木デ樹高 40-50 米=達

* Tristylopsis falcata (Kanemira) Kanehira et Harusima comb. nor. Palaoea falcata Kanehira in Bot. Mag. Tokyo 49 (1935) 271, f. 25. 
スルモノモ稀デナイ。モミ附近ノ低地林二㜪通デアル。

Hibbertia novo-guineensis GIBBS 本屬/植物ハニユージランド及嫁洲/特産卜考

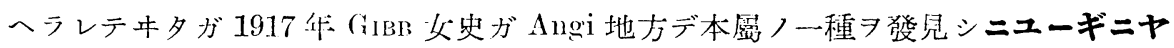

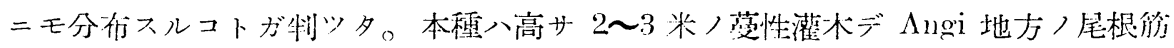
ノ踈林付二通デアル。

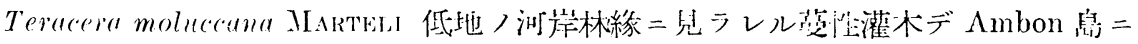
モ分有シテキル。

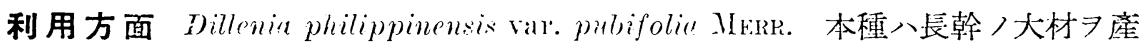

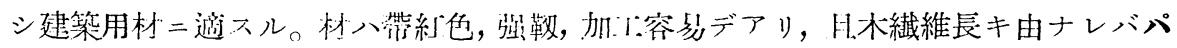
ルプ用沾トシテモ侍望デアラウ。

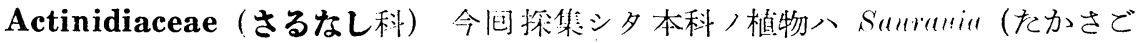

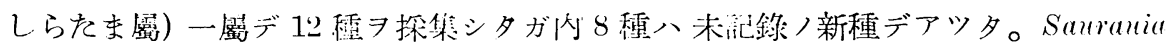

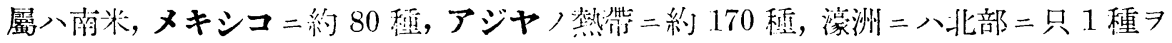
產スルノミデアル。アジヤデハニユーギニヤガ顷モ分化發達シ，ソ/種数モ今可我

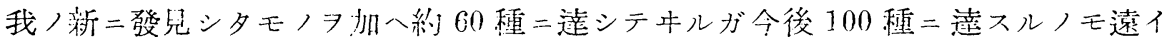
將來デハアルマイ。ニューギニヤデ八低地カラ高地迄分布シテキルガイヅレカト云

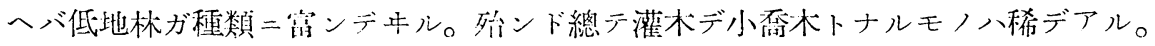

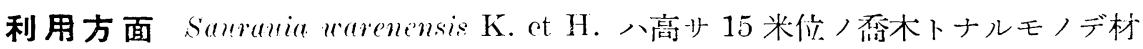
ヨ利用スルコトガ出來ルガ，ソノ他ノ種数八總テ灌朴デ材ノ利用八問题卜サルマイ。

\section{Sapindaceae（むくろじ科）}

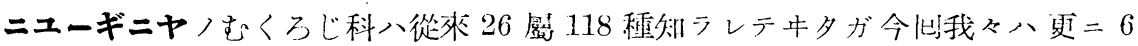

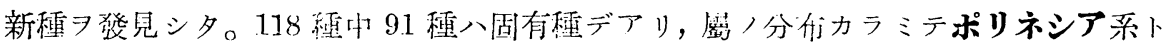

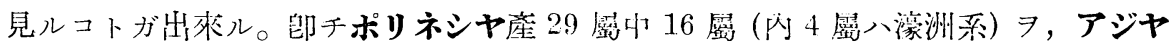
系 37 屬中 9 䪅 Allophylus (1 種), Cupaniopsis (1), Dodonaea (1), Elattostachys (1), Erioglossum (1), Grinoa (2), Harpullia (5), Mischocurpus (2), Pomein (1), Rhysotoechia (2), Curcopterij:x: (1), Toechimu (2), Tristylopsis (1)，即千 13 霓 21 種デアッタ。

今齐屬二就キ略遮スルト次ノ如クナル。

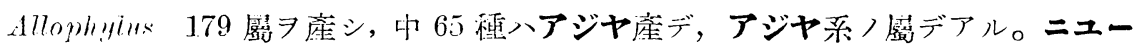

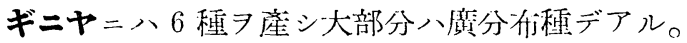

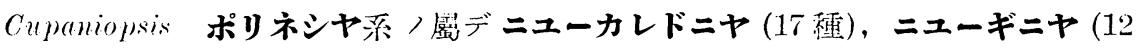
種), 荄洲 (8), フイージー種諸察 (3 種) 二分价シテキル。

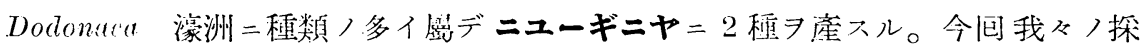

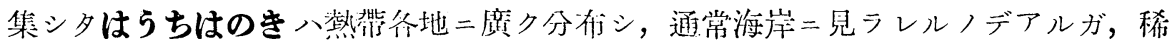
二高地帶二見ラレル。今包探集シタ所八海拔 1900 米モアルAngi 男湖湖㽣ノ第二 林デ殆ンド純林ヨ形成シテキタガ生態熒上梅メテ興味アルコトト思フ。

Elattostachy: ポリネシヤ要素デ 12 種中馬來諸島 $=2$ 種, 濠洲 $=3$ 種 Erioglossum アジヤ要素デ 1 閩 1 種ノ植物デアル。印度, マレーシヤ二廣ク分布 
シニユーギニヤ，濠洲北部，東部迄分死シテキル。

Guioa ポリネシヤ姴素デ，55 種中大部分ハオセアニヤ二分布シ一部八比島迄北 上シテキル。ニューギニヤニハ 14 種习産シ,今包我々ガ發見シタG.amabilis K. et H. 八Angi 地方海拔 2300 米附近ノ屋根二產シ，ニューカレドニヤ産ノ G. venusta RADLK. 二一番近イ種類デアル。

Hurpullia ポリネシヤ要素デニユーギニヤ (17 種), 嫁洲 (7 種), アルー群島 (1), ソロモン群島 (1)，柳印 (1), 此島 (1)，チモール (1)，ニニーカレドニヤ (1)，サモ ア群島 $(1)$ 二分布シテキル。今旬我々ハ 5 種ヨ採集シタガ中 1 種八未記錄ノ新種デ アツ夕。本屬ハニューギニヤ二種類多ク，今後庭二㒄加スルモノト考へル。

Mischocarpu: アジヤ要素デ嫁洲 (5種)，ニューギニヤ (4 種) =及ンデキル。今 间我々ハ2種习採集シタガ，中 M. paradoxus RADLK. 八花ノ標本デアツタガ，實， 標本デ記載シタトラック鼠產, M. Grullauminiana KAN：ト區別ナイ樣デアル。

Rhysotoechia ポリネシヤ要素デ ニューギニヤ (2 種)，滾洲 (4)，豎來諸島 (3)，

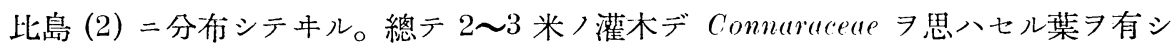

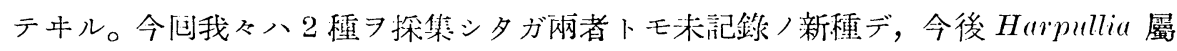
ト共ニニューギニヤデりノ程類八增加スルモノト考へル。

Sarcopteryx ポリネシヤ要素デニユーギニヤ (4 種)，濛洲 (2)，モルッカ群身 (1) 二分布天儿。

Tochima ポリネシヤ妿素デニューギニヤ (3種)，蒃洲 (4) 二分布スル。

Tristylopsis ポリネシヤ姴素デ大平洋，泉二廣ク分布シ北八此身迄來テキル。從 來ニューギニヤ $=3$ 種ヨ產シタガ今包我々八更二一新種 T.noro-gnineensis K. et H.

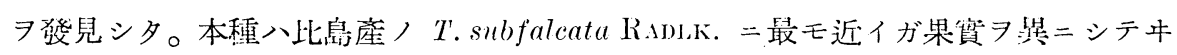

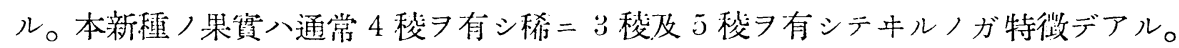

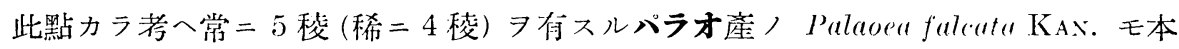
屬ニ移スガ至當デアラウ。

ニニーギニヤノむくろじ科八大部分低地林二限ラレ, 中高地义八高地肃ニハソ， 種類八比較的少イ。Angi 湖方面ノ探集/折モモミ附近/海拔 300 米以上二八殆ン ド本科习見ズ，1900 米附近ノ罗湖附近デ沬うち注のき探集シ，2300 米附近ノ尾根解 ノ森林デ Guioa, Sarcoptery, ヨ犬々一種ヅツ探集シタ狀態デアル。本科ノ植物入-..

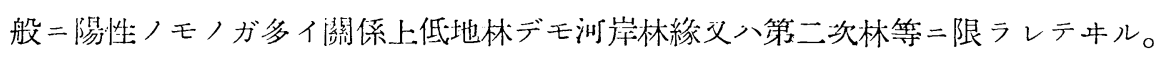

利用方面 Pometia pinnata Forst., Tristylopsis noro-guineensis K. et H. /雨 者八樹高 40 米队外二達シ，通直ナ良材フ產ス儿。殊二前者ハナビレ，モミ方面，低 地林二多產スルノデ将來利用上注月スベキモノト考へル。

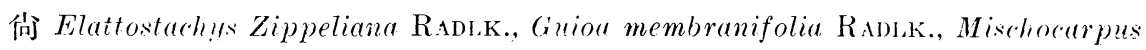
paradcxus RadLK., M. sundaicus BL., Toechima hirsutum RADLK., T. subteres RadLK.,

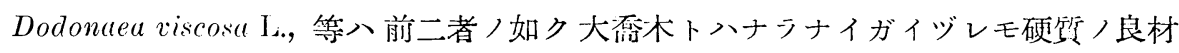
习產スルカラ起築忉其他二利用スルコトガ出來ヤウ。 